\title{
Association of dietary patterns of American adults with bone mineral density and fracture
}

\author{
Mohsen Mazidi ${ }^{1,2, *}$, Andre Pascal Kengne ${ }^{3}$ and Hassan Vatanparast ${ }^{4}$ \\ ${ }^{1}$ Key State Laboratory of Molecular Developmental Biology, Institute of Genetics and Developmental Biology, \\ Chinese Academy of Sciences, Beijing 100101, People's Republic of China: ${ }^{2}$ Institute of Genetics and \\ Developmental Biology, International College, University of Chinese Academy of Science, Beijing, \\ People's Republic of China: ${ }^{3}$ Non-Communicable Disease Research Unit, South African Medical Research Council \\ and University of Cape Town, Cape Town, South Africa: ${ }^{4}$ College Pharmacy \& Nutrition, School of Public Health, \\ Saskatoon SK, Canada
}

Submitted 15 May 2017: Final revision received 16 February 2018: Accepted 23 February 2018; First published online 21 May 2018

\begin{abstract}
Objective: In a representative sample of US adults, we investigated the associations of nutrient patterns (NP) with bone mineral density (BMD) and fractures.

Design: Cross-sectional.

Setting: US community-based National Health and Nutrition Examination Survey (NHANES).

Subjects: Participants with measured data on dietary intake and BMD from 2005 to 2010 were included. Principal components analysis was used to identify NP. BMD was measured using dual-energy X-ray absorptiometry. ANCOVA, adjusted logistic and linear regression models were employed, accounting for the complex survey design and sample weights.

Results: We included a total of 18318 participants, with $47 \cdot 0 \%$ ( $n$ 8607) being men. The mean age was 45.8 years with no sex difference. Three NP emerged, explaining $55.9 \%$ of the variance in nutrient consumption. Multivariable-adjusted linear regressions revealed significant inverse associations between the 'highenergy' NP (rich in carbohydrates and sugar, total fat and saturated fat) and total femur, femoral neck, trochanter and intertrochanter BMD ( $\beta$ coefficient: $-0 \cdot 029$, $-0.025,-0.034$ and -0.021 , respectively, all $P<0 \cdot 001)$, while there were significant associations between the 'nutrient-dense' NP (rich in vitamins, minerals and fibre) and 'healthy fat' NP (high dietary PUFA and MUFA) and BMD at total femur, femoral neck, trochanter and intertrochanter (all $P<0 \cdot 001$ ). In adjusted logistic regression models, the odds of hip, wrist or spine fractures did not vary significantly across NP quartiles.

Conclusions: Nutrient-dense and healthy fat NP are associated with higher BMD at various bone sites, while the high-energy NP is inversely associated with BMD measures.
\end{abstract}

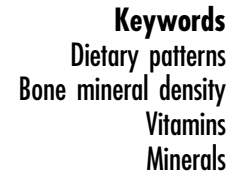

Osteoporosis is a systemic skeletal disease characterized by reduced bone mass and disrupted bone architecture, resulting in increased bone fragility and fracture risk ${ }^{(1)}$. Osteoporosis is commonly referred to as a 'silent disease' because it remains asymptomatic until a fracture occurs. Such osteoporotic fractures are a major cause of morbidity in the elderly population ${ }^{(2)}$. Low bone mineral density (BMD; i.e. number of standard deviations below the mean areal BMD for young adults, $T<-2.5$ ) is marker of osteoporosis and a predictor of low trauma fractures ${ }^{(3)}$.

The accumulation and loss of bone mineral mass is influenced by various factors such as age, sex, ethnicity, heredity, lifestyle (physical activity and smoking) and nutritional status (Ca, protein and vitamin D intakes) ${ }^{(4)}$. Of these, diet is one of the most important modifiable determinants ${ }^{(5)}$. However, most studies have focused on a single nutrient or food/food group to examine the effects on bone health. These common approaches have methodological and conceptual limitations. They can detect the effects of single nutrients or foods on bone health but cannot explain the interactions among nutrients and food items $^{(6)}$. We have comprehensively explained different approaches in evaluating the association between nutrient patterns (NP) and bone health elsewhere ${ }^{(7)}$.

NP analysis has emerged as an alternative approach to overcome the aforementioned limitations in nutritional 
epidemiology ${ }^{(8)}$. In this approach, statistical methods are used to examine the pattern of intake of multiple foods or nutrients and derive single-exposure variables, or $\mathrm{NP}^{(9)}$. Such NP may provide an improved and more generalizable insight into diet-disease relationships ${ }^{(8)}$. Using the NP approach could facilitate the development of public health recommendations that are more convenient to follow $^{(10)}$. Moreover, the human diet includes a range of different food items and complex mixtures of nutrients. Therefore, applying traditional methods (focusing on single nutrients) cannot take into consideration the numerous intercorrelations and interactions among foods and nutrients ${ }^{(8)}$.

Although previous studies have examined the association of NP with BMD, the findings are conflicting. Some studies suggest an inverse relationship between an 'unhealthy' NP (characterized by red and processed meat, fats and sweets) and BMD, and a positive association between a 'healthy' NP (characterized by high consumption of fish, olive oil, fruits and vegetables, and low consumption of red meat and candy) and BMD ${ }^{(11-14)}$. Others have failed to show such associations ${ }^{(7,15)}$. For example, in a sample of 220 adult Greek women, adherence to a Mediterranean dietary pattern was not associated with indices of bone mass ${ }^{(7)}$. Another study of 1928 men and 4611 women from the Canadian Multicentre Osteoporosis Study found no consistent relationship between diet and $\mathrm{BMD}^{(15)}$. Given the inconsistencies across existing studies, we conducted the current study to identify NP associated with high or low BMD at different parts of the body (total femur, femoral neck, intertrochanter, trochanter, Ward's triangle, total spine, lumbar spine L1 to L4), as well as risk of fractures, in a nationally representative sample of US adults. We hypothesized that higher adherence to the Western diet would be associated with a less favourable index of bone health (BMD) and greater likelihood of fractures, while adherence to a healthy diet would be related with favourable bone health and lower risk of fractures.

\section{Methods}

\section{Population}

Data from the National Health and Nutrition Examination Surveys (NHANES) conducted between 2005 and 2010 were used for the present study. NHANES is a repeated cross-sectional survey conducted on an ongoing basis by the US National Center for Health Statistics, applying protocols and procedures described in detail previously ${ }^{(16)}$. All methods were performed in compliance with the Declaration of Helsinki on ethical standards for research involving human subjects ${ }^{(16)}$. NHANES uses a complex, multistage and stratified sampling design to select a sample representative of the civilian and non-institutionalized resident population of the USA. The National Center for Health
Statistics Research Ethics Review Board approved the protocol and all participants provided informed consent ${ }^{(17)}$. Demographic information and interviews were collected using questionnaires administered during home visits, while trained personnel conducted a physical examination and biological sample collection in mobile examination units. The NHANES mobile examination centres are equipped with QDR 4500A fan-beam dual-energy X-ray absorptiometry densitometers (Hologic, Inc.) to measure BMD of the anterior-posterior lumbar spine and proximal femur. Details on measuring the BMD and protocols for the corresponding quality controls can be found elsewhere ${ }^{(18)}$. For fractures, we used data from self-reported hip, wrist (representing distal radius/ulna) and spine fractures ${ }^{(18)}$. A trained phlebotomist drew a blood specimen from the participant's antecubital vein. Detailed information on the measurement of C-reactive protein (CRP) concentrations are available elsewhere ${ }^{(19,20)}$.

Dietary intake was assessed via $24 \mathrm{~h}$ recall by a trained interviewer, during the mobile examination centre visit, by use of a computer-assisted dietary interview system with standardized probes, i.e. the US Department of Agriculture Automated Multiple-Pass Method ${ }^{(21)}$. Briefly, the type and quantity of all foods and beverages consumed in a single $24 \mathrm{~h}$ period before the dietary interview (from midnight to midnight) were collected via the Automated Multiple-Pass Method, which is designed to enhance complete and accurate data collection while reducing the respondent burden $^{(21)}$.

For the present analysis, three survey cycles (i.e. 2005-2006, 2007-2008 and 2009-2010) were combined to produce estimates with greater precision and smaller sampling error. The analytical sample was limited to adults aged $\geq 18$ years. After excluding pregnant and lactating ( $n$ 795) respondents, as well as those with missing information on the variables of interest ( $n$ 1325), the final analytical sample included 18318 respondents from NHANES 2005-2010.

\section{Statistical analysis}

We analysed the data in compliance with prescribed guidelines for analysis of the complex NHANES data set, taking account of the masked variance and utilizing the proposed weighting methodology ${ }^{(22)}$. Factor analysis with orthogonal transformation (Varimax procedure) was applied to derive NP based on the nutrients. We used principal component factor analysis with Varimax orthogonal transformation to generate principal components representative of NP based on the highest correlation coefficients between the nutrients constituting each principal component ${ }^{(23)}$. All the necessary prerequisites of principal component analysis, including linearity, KaiserMeyer-Olkin measure of 0.88 and significant Bartlett's test of sphericity $(P<0 \cdot 001)$, were met. We then used regression methods to calculate the factor scores of each NP for each study participant ${ }^{(23)}$. Factors were retained for 
further analysis based on their natural interpretation and eigenvalues on the scree test ${ }^{(24,25)}$. We computed the factor score for each NP by summing up intakes of nutrients weighted by their factor loadings (see online supplementary material, Supplemental Table 1). Each participant received a factor score for each identified pattern. Simple linear dose-response relationships are unlikely to be found in nutritional epidemiology ${ }^{(26)}$. To avoid issues with departure from a normal distribution and accordingly the distortion of regression coefficients by the extreme values, NP variables were categorized using population-specific quartiles of each NP before inclusion in regression models. The bottom or first quartile of each NP was then used as the reference category in all regression analyses. We computed means of BMD adjusted for age, sex, race/ethnicity, physical activity, smoking and CRP across the quartiles of each NP using ANCOVA. Categorical demographic variables were compared by using ANOVA and $\chi^{2}$ tests, respectively. Fully adjusted multivariable linear regression models (adjusted for age, sex, race/ethnicity, physical activity, smoking and CRP) were used to determine the association of each NP score with BMD. Results were analysed using the complex sample module of the statistical software package IBM SPSS Statistics version 22.0. Sample weights were applied to account for unequal probabilities of selection, non-response bias and oversampling.

\section{Results}

The analytical sample comprised 18318 participants, of whom $47.0 \%$ ( $n$ 8607) were men. The mean age was 45.8 years in the overall sample and did not vary significantly between men and women $(P=0 \cdot 126)$. The White (non-Hispanic) population comprised the majority (69.4\%) of the participants. Furthermore, 56.1\% ( $n$ 8759) of the participants were married and $19.8 \%$ were current smokers $(23.9 \%$ of the men and $16.7 \%$ of the women). Overall, fewer participants engaged in a vigorous physical activity $(5 \cdot 2 \%)$ than in little or no physical activity (24.0\%). As can be seen from Table 1, there were significant differences across quartiles of each NP with respect to race/ethnicity, education and sex distribution $(P<0.001$ for all comparisons). For each NP, participants in the upper higher quartile were younger than those in the bottom quartile $(P<0 \cdot 001$ for all comparisons). Furthermore, participants in the upper quartile of the first NP had a higher level of CRP compared with those in the bottom quartile $(0.45 v .0 .36 \mathrm{mg} / \mathrm{dl}, P<0.001)$, while participants in the upper quartile of the second and third NP had lower levels of CRP compared with those in the bottom quartile (both $P<0 \cdot 001$; Table 1).

The principal component method uncovered three NP altogether explaining $55.9 \%$ of the variance in consumption of dietary nutrients. The first NP was essentially representative of a diet high in carbohydrates and sugar, total fat

Table 1 Demographic characteristics of participants across quartiles of nutrient patterns (NP): US adults aged $\geq 18$ years ( $n$ 18318), National Health and Nutrition Examination Survey (NHANES) 2005-2010

\begin{tabular}{|c|c|c|c|c|c|c|c|c|c|c|c|c|c|c|c|}
\hline \multirow[b]{3}{*}{ Characteristic } & \multicolumn{4}{|c|}{$\begin{array}{c}\text { Quartile of 'high-energy' } \\
\text { NP }\end{array}$} & \multirow[b]{3}{*}{$P$ value } & \multicolumn{4}{|c|}{$\begin{array}{c}\text { Quartile of 'nutrient-dense' } \\
\text { NP }\end{array}$} & \multicolumn{5}{|c|}{$\begin{array}{c}\text { Quartile of 'healthy fat' } \\
\text { NP }\end{array}$} & \multirow[b]{3}{*}{$P$ value } \\
\hline & Q1 & Q2 & Q3 & Q4 & & Q1 & Q2 & Q3 & Q4 & \multirow[b]{2}{*}{$P$ value } & Q1 & Q2 & Q3 & Q4 & \\
\hline & $\%$ & $\%$ & $\%$ & $\%$ & & $\%$ & $\%$ & $\%$ & $\%$ & & $\%$ & $\%$ & $\%$ & $\%$ & \\
\hline \multicolumn{16}{|l|}{ Sex } \\
\hline Male & 31.1 & 41.1 & 51.4 & 71.1 & \multirow[t]{2}{*}{$<0.001$} & 31.9 & $41 \cdot 8$ & 51.4 & $69 \cdot 6$ & \multirow{2}{*}{$<0.001$} & 30.7 & 41.6 & $51 \cdot 1$ & $71 \cdot 1$ & \multirow[t]{2}{*}{$<0.001$} \\
\hline Female & 68.9 & 58.9 & 48.6 & 28.9 & & $68 \cdot 1$ & $58 \cdot 2$ & $48 \cdot 6$ & $30 \cdot 4$ & & $69 \cdot 3$ & $58 \cdot 4$ & 48.9 & 28.9 & \\
\hline \multicolumn{16}{|l|}{ Race/ethnicity } \\
\hline Mexican-American & 20.5 & $19 \cdot 6$ & $19 \cdot 0$ & $17 \cdot 3$ & \multirow[t]{5}{*}{$<0.001$} & 19.6 & $19 \cdot 3$ & 18.9 & 18.5 & \multirow[t]{5}{*}{$<0.001$} & $17 \cdot 4$ & $18 \cdot 4$ & $19 \cdot 3$ & $21 \cdot 1$ & \multirow[t]{5}{*}{$<0.001$} \\
\hline Other Hispanic & $10 \cdot 2$ & $8 \cdot 6$ & 7.5 & $7 \cdot 2$ & & $9 \cdot 6$ & $8 \cdot 7$ & 7.9 & $7 \cdot 3$ & & $10 \cdot 0$ & $8 \cdot 8$ & $7 \cdot 7$ & $7 \cdot 0$ & \\
\hline White (non-Hispanic) & 42.5 & 48.0 & 48.6 & 49.5 & & 38.9 & $46 \cdot 9$ & $49 \cdot 8$ & 53.1 & & 40.9 & 47.6 & $49 \cdot 2$ & 50.9 & \\
\hline Non-Hispanic Black & $22 \cdot 8$ & $19 \cdot 8$ & $20 \cdot 0$ & $21 \cdot 0$ & & $27 \cdot 6$ & $21 \cdot 0$ & $18 \cdot 8$ & $16 \cdot 3$ & & $26 \cdot 8$ & $20 \cdot 5$ & $19 \cdot 3$ & $17 \cdot 1$ & \\
\hline Others & $4 \cdot 1$ & 4.0 & 4.9 & $5 \cdot 0$ & & 4.4 & $4 \cdot 2$ & 4.6 & 4.8 & & 4.9 & 4.6 & 4.6 & 3.9 & \\
\hline \multicolumn{16}{|l|}{ Marital status } \\
\hline Married & $45 \cdot 3$ & 47.6 & $53 \cdot 1$ & $52 \cdot 8$ & \multirow[t]{3}{*}{$<0.001$} & 44.5 & $51 \cdot 2$ & $55 \cdot 1$ & $54 \cdot 5$ & \multirow[t]{3}{*}{$<0.001$} & $45 \cdot 9$ & $51 \cdot 2$ & $54 \cdot 3$ & $54 \cdot 1$ & \multirow[t]{3}{*}{$<0.001$} \\
\hline Widowed & $15 \cdot 3$ & 13.7 & $6 \cdot 7$ & 3.2 & & $12 \cdot 3$ & 9.1 & 7.5 & 4.5 & & $13 \cdot 2$ & 9.5 & 7.4 & 3.4 & \\
\hline Divorced & $9 \cdot 3$ & 11.7 & $10 \cdot 9$ & 8.4 & & $11 \cdot 8$ & $11 \cdot 0$ & $9 \cdot 3$ & $9 \cdot 0$ & & 11.5 & $10 \cdot 8$ & $10 \cdot 1$ & $8 \cdot 8$ & \\
\hline \multicolumn{16}{|l|}{ Age (years) } \\
\hline Mean & $52 \cdot 1$ & $49 \cdot 4$ & $46 \cdot 6$ & 41.9 & \multirow[t]{2}{*}{$<0.001$} & $49 \cdot 2$ & $49 \cdot 0$ & $47 \cdot 7$ & 43.6 & \multirow[t]{2}{*}{$<0.001$} & $50 \cdot 1$ & $49 \cdot 6$ & $47 \cdot 2$ & 42.5 & \multirow[t]{2}{*}{$<0.001$} \\
\hline SE & 0.2 & 0.6 & 0.2 & $0 \cdot 1$ & & 0.2 & 0.1 & 0.5 & 0.6 & & 0.2 & 0.2 & 0.1 & 0.3 & \\
\hline \multicolumn{16}{|c|}{ C-reactive protein $(\mathrm{mg} / \mathrm{dl})$} \\
\hline Mean & 0.36 & 0.37 & 0.42 & 0.45 & \multirow[t]{2}{*}{$<0.001$} & 0.44 & 0.39 & 0.38 & 0.35 & \multirow[t]{2}{*}{$<0.001$} & 0.43 & 0.41 & 0.38 & 0.37 & \multirow[t]{2}{*}{$<0.001$} \\
\hline SE & 0.01 & 0.02 & 0.01 & 0.01 & & 0.03 & 0.02 & 0.01 & 0.01 & & 0.01 & 0.01 & 0.02 & 0.01 & \\
\hline BMI $\left(\mathrm{kg} / \mathrm{m}^{2}\right)$ & 28.8 & 28.6 & 28.7 & $28 \cdot 8$ & 0.326 & 29.0 & $28 \cdot 8$ & $28 \cdot 6$ & $28 \cdot 4$ & 0.098 & $29 \cdot 1$ & 28.8 & 28.7 & $28 \cdot 7$ & 0.342 \\
\hline Fractures in hip & $0 \cdot 2$ & $0 \cdot 6$ & 0.3 & $0 \cdot 3$ & 0.235 & 0.5 & 0.3 & 0.2 & 0.4 & 0.125 & 0.4 & $0 \cdot 2$ & 0.3 & 0.5 & 0.098 \\
\hline Fractures in wrist & 1.6 & $3 \cdot 2$ & $2 \cdot 9$ & 1.2 & $0 \cdot 101$ & $2 \cdot 1$ & 3.0 & 1.6 & $2 \cdot 2$ & 0.082 & 2.9 & 1.6 & $2 \cdot 3$ & $2 \cdot 1$ & 0.123 \\
\hline Fractures in spine & 0.6 & 0.7 & 0.6 & 0.4 & 0.253 & 0.5 & 0.8 & 0.5 & 0.5 & 0.182 & 0.6 & 0.5 & 0.6 & 0.6 & 0.428 \\
\hline
\end{tabular}

Data are presented as percentages or as means with their standard errors where noted. ANOVA or the $\chi^{2}$ test was applied. 
and saturated fat (called the 'high-energy' NP); the second NP was high in vitamins, minerals and fibre ('nutrient-dense' $\mathrm{NP}$ ) and the third NP was mainly representative of high dietary PUFA and MUFA ('healthy fat' NP).

The age, sex, race/ethnicity, physical activity, smoking and CRP-adjusted means of BMD for different sites across quartiles of each NP are shown in Table 2. BMD at the total femur, femoral neck, trochanter and intertrochanter decreased significantly with increasing quartile of the high-energy NP $(P<0.001$ for all). BMD at the total femur, femoral neck and intertrochanter increased significantly across increasing quartiles of the nutrient-dense and healthy fat NP (all $P<0 \cdot 001$ ). The profile of the associations was similar in age, sex, race/ethnicity, physical activity, smoking, CRP, BMI and hormone replacement therapy-adjusted linear regression models examining the continuous associations of NP with BMD. Indeed, there was a significant inverse association between the highenergy NP and total femur $(\beta=-0.029)$, femoral neck $(\beta=-0.025)$ and trochanter BMD $(\beta=-0.034$; all $P<0.001)$. On the other hand, there was a significant positive association between the nutrient-dense and healthy fat NP and BMD at the total femur, femoral neck, trochanter and intertrochanter (all $P<0.001$; Table 2). No significant interactions were found between NP (all interaction $P>0 \cdot 153$ ).

Percentage of fractures in the hip, wrist and spine were $1.4,8.9$ and $2.3 \%$, respectively. The rate of fracture by quartile of each NP is shown in Table 1. In logistic regression models adjusted for age, sex, race/ethnicity, physical activity, smoking, CRP, BMI and hormone replacement therapy, there was no significant variation across quartile of NP in the odds of fractures in the hip, wrist and spine (Table 3).

\section{Discussion}

Findings from the present study revealed that BMD at different sites of the proximal femur was inversely associated with a diet consisting highly of carbohydrates and sugar, total fat and saturated fat, and directly associated with a diet comprising vitamins, minerals and fibre. No association was found between NP and fractures, a finding to be interpreted in the context of a considerably low number of fractures.

In agreement with our results, several previous studies have demonstrated the direct association of fruits and vegetables (main sources of fibre, minerals and vitamins in the diet) with bone health ${ }^{(11,12,14,15)}$. The intake of fruits and vegetables in combination with fish was associated with high BMD in Japanese female farmers ${ }^{(12)}$. The existing Mediterranean diet score (MDS) was shown to be associated with high $\mathrm{BMD}^{(27)}$. Studies on the MDS and fracture risk have reported both unfavourable ${ }^{(28)}$ and favourable ${ }^{(29)}$ associations. The Mediterranean diet is

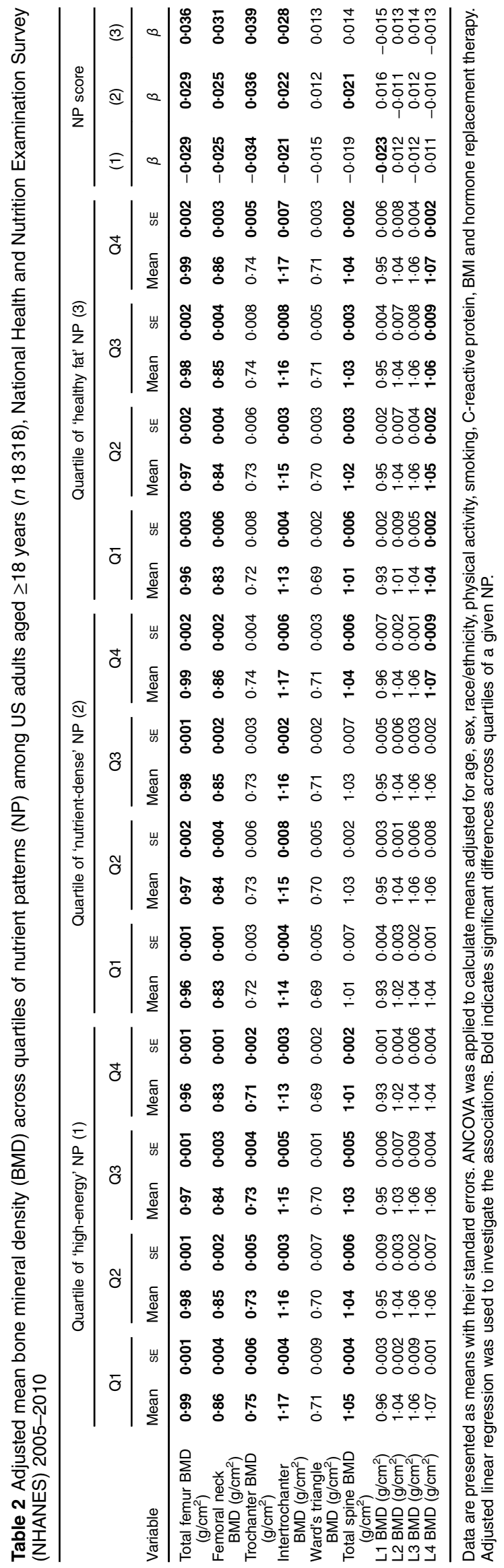


known for its high content of cereals, legumes, fruits, nuts, vegetables, oils and fish, as well as low content of dairy and meat products. Although none of the patterns in our analyses of NHANES data exactly represents the Mediterranean diet, there are similarities with the nutrientdense NP observed in our study, considering its high factor loadings for vitamins, minerals and fibre. Fruits and vegetables may act on bones by providing base buffers against excess dietary metabolic acids, increasing osteoclast activity that promotes bone resorption ${ }^{(30)}$ and balancing the $\mathrm{pH}$ of body fluids to the physiological range $^{(31)}$. In the Women's Health Initiative study, a low-fat and high-fruit, -vegetable and -grain diet intervention slightly decreased the risk of multiple falls and marginally lowered hip BMD in postmenopausal women ${ }^{(32)}$. However, Pedone et al. reported no relationship between dietary acid load and BMD in elderly women ${ }^{(33)}$. In a prospective study among 15572 adults included in the China Health and Nutrition Survey, two dietary (traditional and modern) and two nutrient (plant- and animal-sourced) patterns were identified. During followup, participants in the upper tertile of the modern dietary and animal-sourced patterns had a $34 \%$ (hazard ratio= $1.34 ; 95 \%$ CI 1.06, 1.71) and $37 \%$ (hazard ratio $=1.37$; $95 \%$ CI $1.08,1.72)$ increase in fracture risk compared with those in the first tertile, respectively ${ }^{(34)}$. In another study evaluating the association between NP and BMD among elderly Australians, animal- and plant-sourced patterns were not associated with BMD, while a mixed-source pattern had protective effect on BMD loss ${ }^{(35)}$.

After adjustment for CRP we found that there was still a significant relationship between $\mathrm{BMD}$ and $\mathrm{NP}$, suggesting an independent role of diet on bone health. Studies have reported evidence on the role of inflammatory factors on bone remodelling. For example, in a study using NHANES III data (2807 females aged 65 years or older), there was an association between CRP and $\mathrm{BMD}^{(36)}$. Moreover, in another prospective study (1872 communitydwelling women), fracture risk increased with increasing CRP levels ${ }^{(37)}$.

Diets rich in minerals and vitamins are positively associated with $\mathrm{BMD}^{(38)}$. The nutrient-dense pattern in our study represented minerals and vitamins that are beneficial for the fundamental architecture of bone ${ }^{(39,40)}$. The favourable effects of $\mathrm{Ca}$ and dairy food on bone health have been well established ${ }^{(39,40)}$. Minerals found in fruits and vegetables might contribute to healthy bone remodelling via a favourable impact on osteoblastic and osteoclastic activity. K and Mg may contribute to the acidbase balance in the body, as discussed before, and prevent bone loss. $\mathrm{K}$ could also increase the retention of $\mathrm{Ca}$ in the kidneys, independently of its role in the alkaline state of the body. Mg is also necessary for Ca metabolism. Vitamin $\mathrm{C}$ may affect bone health through its antioxidant properties, which suppress osteoclast activity ${ }^{(41)}$. It also acts as a cofactor for osteoblast differentiation and collagen 
formation $^{(41)}$. Carotenoids and other antioxidants also affect bone health by reducing oxidative stress ${ }^{(41)}$. Vitamin $\mathrm{K}$ is involved in bone matrix formation, where mineralization happens ${ }^{(42)}$.

We identified a pattern characterized by high consumption of carbohydrates, sugar, total fat and saturated fat, the high-energy NP, which is similar to ones identified in other studies ${ }^{(11,14,15)}$. McNaughton and co-workers ${ }^{(14)}$ identified a 'high-energy nutrient-poor pattern' characterized by high intakes of refined cereals, soft drinks, fried potatoes, processed meat, beer, chocolate, confectionery and added sugar, and low consumption of vegetables, fruits and wholegrain cereals, which was significantly inversely associated with total body BMD. Similarly, for the 'candy pattern' observed by Tucker et $a l .{ }^{(11)}$ in the Framingham Cohort Study, a diet rich in refined foods and lacking in nutrient-dense foods may be detrimental to bone health in men. Indeed, participants in the 'candy' cluster had a lower BMD in comparison with individuals in the 'fruit, vegetables and cereal' and 'alcohol' clusters ${ }^{(11)}$. Furthermore, a recent study from Brazil found that a 'sweet foods, coffee and tea pattern' was inversely correlated with $\mathrm{BMD}^{(43)}$. Additionally, like the high-energy $\mathrm{NP}$ in our study, high intakes of fat and saturated fat showed a borderline inverse association with BMD among Japanese women $^{(12)}$.

Fish and seafood are rich in PUFA, especially $n-3$ fatty acids, which are known to have an anti-inflammatory impact that benefits bone ${ }^{(44)}$. We found the healthy fat NP to be associated with BMD in adults. A systematic review of ten randomized controlled trials revealed that $n-3$ and n-6 fatty acids when combined with Ca or dairy products had a significant impact on bone measures in some but not all trials ${ }^{(45)}$. The association between $n-3$ fatty acids and bone biomarkers and BMD could be explained by their anti-inflammatory effect, although more studies are needed to clarify the potential mechanisms.

There are several limitations to the present study. First, the results from our cross-sectional study, although nationally representative, cannot demonstrate a causal relationship. Second, although our analysis included known potential variables that can affect bone health in terms of environmental and genetic factors, residual confounding variables may still exist. We used a wide age range in adulthood that might not be the best approach in evaluating the association between NP and BMD. In an ideal situation, when sample size allows, women after menopause should be studied separately controlling for other potential covariates such as hormone replacement therapy. The statistical power was low to reliably investigate the association between NP and fracture. Moreover, fracture cases were assessed based on self-reports.

The study has several strengths. We had a sample selected randomly from the general US population. Therefore, the results obtained from this nationally representative sample can be extrapolated to the entire population. In addition, a large number of participants aged 18-80 years, the use of standardized procedures and the inclusion of both men and women are other important strengths.

A healthy nutrient-dense NP, characterized by high intakes of minerals, vitamins and fibre, may benefit BMD independent of potential confounding factors. In contrast, adherence to a high-energy NP characterized by high consumption of total and saturated fats, carbohydrates and sugar may pose a risk for low BMD.

\section{Acknowledgements}

Financial support: This research received no specific grant from any funding agency in the public, commercial or not-for-profit sectors. M.M. was supported by a TWAS studentship of the Chinese Academy of Sciences. Conflict of interest: None. Authorship: M.M. analysed and interpreted the data and drafted the manuscript. A.P.K. conceived of and designed the study, and carried out critical revision of the manuscript. H.V. conceived of and designed the study, and carried out critical revision of the manuscript. Ethics of buman subject participation: NHANES was performed in compliance with the Declaration of Helsinki and all protocols involving human subjects were approved by the National Center for Health Statistics Research Ethics Review Board. Informed consent was obtained from all participants.

\section{Supplementary material}

To view supplementary material for this article, please visit https://doi.org/10.1017/S1368980018000939

\section{References}

1. Anon. (1993) Consensus development conference: diagnosis, prophylaxis, and treatment of osteoporosis. Am JMed 94, 646-650.

2. Plawecki K \& Chapman-Novakofski K (2010) Bone health nutrition issues in aging. Nutrients 2, 1086-1105.

3. Hernlund E, Svedbom A, Ivergård M et al. (2013) Osteoporosis in the European Union: medical management, epidemiology and economic burden. Arch Osteoporos 8, 136.

4. Cooper C, Westlake S, Harvey N et al. (2006) Review: developmental origins of osteoporotic fracture. Osteoporos Int 17, 337-347.

5. Kitchin B \& Morgan S (2003) Nutritional considerations in osteoporosis. Curr Opin Rheumatol 15, 476-480.

6. Movassagh EZ \& Vatanparast H (2017) Current evidence on the association of dietary patterns and bone health: a scoping review. Adv Nutr 8, 1-16.

7. Kontogianni MD, Melistas L, Yannakoulia $\mathrm{M}$ et al. (2009) Association between dietary patterns and indices of bone mass in a sample of Mediterranean women. Nutrition 25, 165-171.

8. Hu FB (2002) Dietary pattern analysis: a new direction in nutritional epidemiology. Curr Opin Lipidol 13, 3-9.

9. Newby PK \& Tucker KL (2004) Empirically derived eating patterns using factor or cluster analysis: a review. Nutr Rev 62, 177-203. 
10. Slattery ML (2008) Defining dietary consumption: is the sum greater than its parts? Am J Clin Nutr 88, 14-15.

11. Tucker KL, Chen H, Hannan MT et al. (2002) Bone mineral density and dietary patterns in older adults: the Framingham Osteoporosis Study. Am J Clin Nutr 76, 245-252.

12. Okubo H, Sasaki S, Horiguchi H et al. (2006) Dietary patterns associated with bone mineral density in premenopausal Japanese farmwomen. Am J Clin Nutr 83, 1185-1192.

13. Hardcastle A, Aucott L, Fraser W et al. (2011) Dietary patterns, bone resorption and bone mineral density in early postmenopausal Scottish women. Eur J Clin Nutr 65, 378-385.

14. McNaughton SA, Wattanapenpaiboon N, Wark JD et al. (2011) An energy-dense, nutrient-poor dietary pattern is inversely associated with bone health in women. $J$ Nutr 141, 1516-1523.

15. Langsetmo L, Poliquin S, Hanley DA et al. (2010) Dietary patterns in Canadian men and women ages 25 and older: relationship to demographics, body mass index, and bone mineral density. BMC Musculoskelet Disord 11, 20.

16. Kassebaum N, Kyu HH, Zoeckler L et al. (2017) Child and adolescent health from 1990 to 2015: findings from the Global Burden of Diseases, Injuries, and Risk Factors 2015 Study. JAMA Pediatr 171, 573-592.

17. Mazidi M, Michos ED \& Banach M (2017) The association of telomere length and serum 25-hydroxyvitamin D levels in US adults: the National Health and Nutrition Examination Survey. Arch Med Sci 13, 61-65.

18. Cogswell ME, Looker AC, Pfeiffer CM et al. (2009) Assessment of iron deficiency in US preschool children and nonpregnant females of childbearing age: National Health and Nutrition Examination Survey 2003-2006. Am J Clin Nutr 89, $1334-1342$.

19. Mazidi M, Kengne AP, Mikhailidis DP et al. (2018) Effects of selected dietary constituents on high-sensitivity C-reactive protein levels in US adults. Ann Med 50, 1-6.

20. Mazidi M, Gao HK, Vatanparast $\mathrm{H}$ et al. (2017) Impact of the dietary fatty acid intake on C-reactive protein levels in US adults. Medicine (Baltimore) 96, e5736.

21. Ahluwalia N, Dwyer J, Terry A et al. (2016) Update on NHANES dietary data: focus on collection, release, analytical considerations, and uses to inform public policy. $A d v$ Nutr 7, 121-134.

22. Mazidi M, Kengne AP, Mikhailidis DP et al. (2017) Dietary food patterns and glucose/insulin homeostasis: a crosssectional study involving 24,182 adult Americans. Lipids Health Dis 16, 192.

23. Mazidi M \& Kengne AP (2017) Nutrient patterns and their relationship with general and central obesity in US adults. Eur J Clin Invest. Published online: 10 March 2017. doi: $10.1111 /$ eci.12745.

24. Stanhope KL (2012) Role of fructose-containing sugars in the epidemics of obesity and metabolic syndrome. Annu Rev Med 63, 329-343.

25. Khayyatzadeh SS, Moohebati M, Mazidi M et al. (2016) Nutrient patterns and their relationship to metabolic syndrome in Iranian adults. Eur J Clin Invest 46, 840-852.

26. Willett W (2013) Nutritional Epidemiology, 3rd ed. New York: Oxford University Press.

27. Rivas A, Romero A, Mariscal-Arcas M et al. (2013) Mediterranean diet and bone mineral density in two age groups of women. Int J Food Sci Nutr 64, 155-161.
28. Feart C, Lorrain S, Coupez VG et al. (2013) Adherence to a Mediterranean diet and risk of fractures in French older persons. Osteoporos Int 24, 3031-3041.

29. Benetou V, Orfanos P, Pettersson-Kymmer U et al. (2013) Mediterranean diet and incidence of hip fractures in a European cohort. Osteoporos Int 24, 1587-1598.

30. Arnett TR \& Dempster DW (1986) Effect of pH on bone resorption by rat osteoclasts in vitro. Endocrinology 119, 119-124.

31. Bushinsky DA (1996) Metabolic alkalosis decreases bone calcium efflux by suppressing osteoclasts and stimulating osteoblasts. Am J Physiol 271, F216-F222.

32 McTiernan A, Wactawski-Wende J, Wu L et al. (2009) Low-fat, increased fruit, vegetable, and grain dietary pattern, fractures, and bone mineral density: the Women's Health Initiative Dietary Modification Trial. Am J Clin Nutr 89, 1864-1876.

33. Pedone C, Napoli N, Pozzilli P et al. (2010) Quality of diet and potential renal acid load as risk factors for reduced bone density in elderly women. Bone 46, 1063-1067.

34. Mangano KM, Sahni S, Kiel DP et al. (2017) Dietary protein is associated with musculoskeletal health independently of dietary pattern: the Framingham Third Generation Study. Am J Clin Nutr 105, 714-722.

35. Melaku YA, Gill TK, Taylor AQ et al. (2017) Association between nutrient patterns and bone mineral density among ageing adults. Clin Nutr ESPEN 22, 97-106.

36. Ganesan K, Teklehaimanot S, Tran TH et al. (2005) Relationship of C-reactive protein and bone mineral density in community-dwelling elderly females. J Natl Med Assoc 97, 329-333.

37. Ishii S, Cauley JA, Greendale GA et al. (2013) C-reactive protein, bone strength, and nine-year fracture risk: data from the Study of Women's Health Across the Nation (SWAN). J Bone Miner Res 28, 1688-1698.

38. Whisner CM \& Castillo LF (2018) Prebiotics, bone and mineral metabolism. Calcif Tissue Int 102, 443-479.

39. Nordin B (2009) The effect of calcium supplementation on bone loss in 32 controlled trials in postmenopausal women. Osteoporos Int 20, 2135-2143.

40. Tang BM, Eslick GD, Nowson C et al. (2007) Use of calcium or calcium in combination with vitamin D supplementation to prevent fractures and bone loss in people aged 50 years and older: a meta-analysis. Lancet 370, 657-666.

41. Gabbay KH, Bohren KM, Morello R et al. (2010) Ascorbate synthesis pathway: dual role of ascorbate in bone homeostasis. J Biol Chem 285, 19510-19520.

42. Fusaro M, Mereu MC, Aghi A et al. (2017) Vitamin K and bone. Clin Cases Miner Bone Metab 14, 200-206.

43. De França N, Camargo M, Lazaretti-Castro M et al. (2016) Dietary patterns and bone mineral density in Brazilian postmenopausal women with osteoporosis: a crosssectional study. Eur J Clin Nutr 70, 85-90.

44. Mazidi M, Shivappa N, Wirth MD et al. (2017) The association between dietary inflammatory properties and bone mineral density and risk of fracture in US adults. Eur J Clin Nutr 71, 1273-1277.

45. Orchard TS, Pan X, Cheek F et al. (2012) A systematic review of omega-3 fatty acids and osteoporosis. Br J Nutr 107, Suppl. 2, S253-S260. 